\title{
Control of COVID-19 in Australia through quarantine: the role of Special Health Accommodation (SHA) in New South Wales, Australia
}

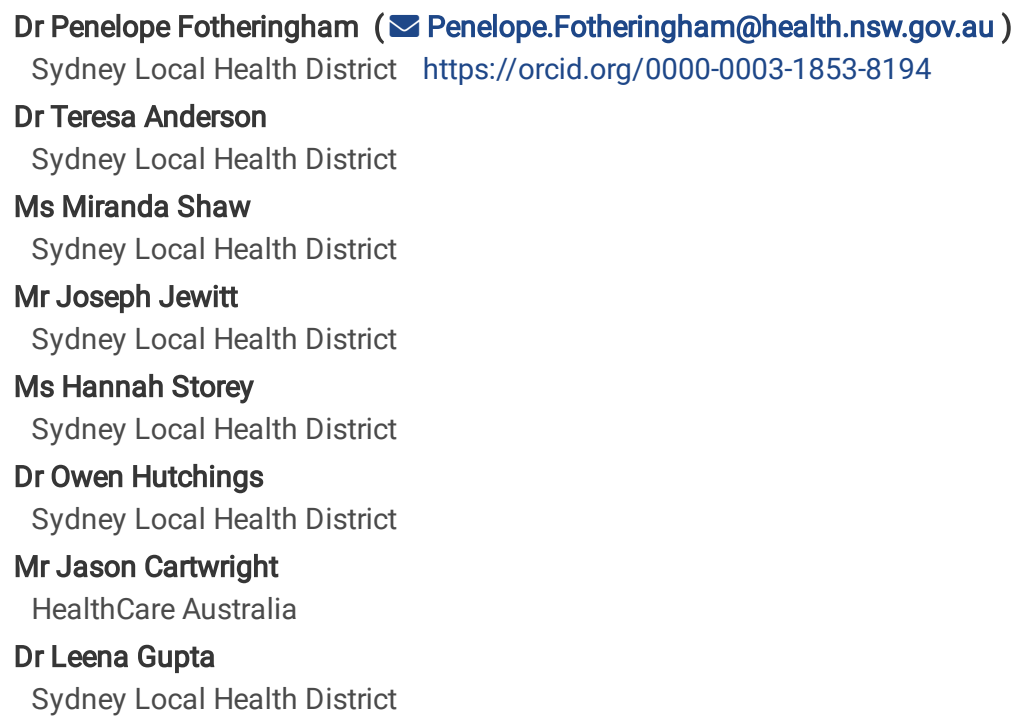

\section{Research Article}

Keywords: COVID-19, Quarantine, Public Health, Telemedicine, Virtual Health care

Posted Date: June 11th, 2020

DOI: https://doi.org/10.21203/rs.3.rs-34542/v1

License: @ (i) This work is licensed under a Creative Commons Attribution 4.0 International License. Read Full License

Version of Record: A version of this preprint was published at BMC Public Health on January 27th, 2021. See the published version at https://doi.org/10.1186/s12889-021-10244-7. 


\section{Abstract}

\section{Background}

The first COVID-19 cases were diagnosed in Australia on 25 January 2020. Initial epidiemiology showed that the majority of cases were in returned travellers from overseas. One aspect of Public Health response was to introduce compulsory 14 day quarantine for all travellers returning to NSW by air or sea in Special Health Accommodation (SHA).

\section{Methods}

The SHA was established with a comprehensive governance structure, clinical management through rpavirtual hospital remote management and site management with health care workers, NSW Police and accommodation staff.

\section{Findings}

From 29 March to 29 April 2020, 373 returned travellers were admitted to the SHA from Sydney Airport. 88 (26.1\%) of those swabbed were positive for SARS-CoV $2,0.91 \%$ of all returning air travellers. The day of diagnosis of COVID-19 varied from Day 1 to Day 13 , with $63.6 \%$ ( $n=56$ ) of these in the first week of quarantine. $50 \%$ of the people in the SHA were referred to rpavirtual for ongoing clinical management. 7 patients required admission to hospital for ongoing clinical care.

\section{Interpretation}

The Public Health response to COVID-19 in Australia included early and increased case detection through testing, tracing of contacts of confirmed cases, social distancing and prohibition of gatherings. In addition to these measures, the introduction of mandated self-quarantine for travellers to Australia was integral to the successful containment of COVID-19 in NSW and in Australia through the prevention of transmission locally and interstate from returning travellers.

\section{Introduction}

The principles of quarantine date back to the $14^{\text {th }}$ century in Italy, when ships arriving in Venice were required to anchor for 40 days prior to landing to ensure that there were no cases of the plague on board.(1) Quarantine refers to the separation of those that have been exposed to a disease but are not yet symptomatic from others who are susceptible to the disease (close contacts). Isolation is another aspect of quarantine, where people who have been diagnosed with the disease (cases) are separated from those who are not infected. The aim is to limit or prevent the spread of disease into a broader population.

Due to the advent of effective disease prevention with vaccination and effective medical treatment, quarantine became less of a focus in the modern world for communicable disease prevention and control. Widespread use of quarantine in Australia has not occurred since the Influenza pandemic of 1918, when Maritime quarantine commenced on 17 October 1918 prior to the first cases of influenza in January 1919. (2, 3) Internationally quarantine has been used in localised regions with the Severe Acute Respiratory Syndrome (SARS) and Middle East Respiratory Syndrome (MERS) epidemics. The use of quarantine on a global scale has not been seen prior to the current SARS-CoV-2 Pandemic that emerged in Wuhan, China in December 2019.

The first COVID-19 cases in Australia were diagnosed on 25 January 2020 in Victoria and NSW. By the end of March 2020 there were 2182 confirmed cases in NSW from 217030 tests performed.(4) Initial epidemiology showed that the majority of confirmed cases were amongst recent arrivals from overseas and their contacts.(5) A major feature of Australia's public health response to SARS-CoV-2 has been to restrict population movements especially through international travel with the use of legislated quarantine. In addition to this, other public health control measures have been implemented such as early and increased case detection through testing, tracing of contacts of confirmed cases, social distancing and prohibition of gatherings. $(6,7)$ These measures have been combined in the Special Health Accommodation (SHA) that provides a quarantine environment with the ability to test suspected cases, clinically manage suspected and confirmed cases and cohort individuals in a supportive setting. This paper describes the new approach to quarantine in the SHA, the first Australian "health hotel" for isolation, quarantine and clinical management.

\section{Quarantine in NSW, Australia}

The NSW Government issued multiple Orders under the Public Health Act regarding COVID-19 restricting movement of returned travellers. The first order, on 17 March, 2020, mandated self-quarantine for 14 days for any returned travellers to NSW. (8) Australian borders were closed to non-citizens and residents from 9pm AEDT Friday, 20 March, and a further order, on 29 March, enforced mandatory quarantine in a quarantine or medical facility for 14 days after arriving in NSW by air or sea. $(9,10)$

When the mandatory quarantine order was enacted on 29 March, Sydney Local Health District (SLHD) extended an existing health accommodation service to establish the Special Health Accommodation (SHA), a unique service designed to support those affected by the NSW public health 
response to COVID-19. The existing service had been responsible for housing the majority of displaced people in NSW who could not effectively quarantine and isolate at home following earlier COVID-19 Public Health Orders $(6,9)$. It was broadened to include those now subject to the Public Health Orders (COVID-19 Air Transportation Quarantine and COVID-19 Maritime Quarantine) that mandated quarantine in a quarantine or medical facility for returned travellers.

\section{Methods}

\section{Establishment of the NSW Special Health Accommodation service}

Since 29 March 2020 returning travellers to NSW have been assessed and triaged for allocation to appropriate accommodation by trained NSW Health officials. Returned travellers are triaged for accommodation according to their state of health on arrival. Asymptomatic returned travellers are managed in accommodation for quarantine that is run by NSW Police and supported by the NSW State Health Emergency Operations Centre (SHEOC) and Health Care Australia. Returning travellers symptomatic of an influenza-like-illness (ILI) are tested for COVID-19 with a nasopharyngeal swab through polymerase chain reaction (PCR) to detect the SARS-CoV-2 nucleic acid (RNA). Returning travellers are appropriately isolated until their results return. Any person that is a possible or suspected case of COVID-19 that is medically stable is isolated in the NSW SHA. The triage process for allocation to these services from arrival is outlined in Figure 1.

The accommodation comprises five repurposed hotel facilities that have been negotiated for use by the SLHD. There is a 356 room capacity and the mix of facilities (outlined in Table 1) was specifically designed to ensure that the needs of all different family groups could be appropriately managed. This service is unique in the state and is able to house any person who enters Australia through NSW. This includes Australian citizens, permanent residents and overseas residents who are returning to Australia. Patients admitted to the SHA are required to be ambulant, have the capacity to manage their own medications and any pre-existing conditions should be medically stable. On arrival at the SHA the patients are met by a nurse in personal protective equipment (PPE) and are then escorted and orientated to their accommodation.

\begin{tabular}{|lllll|}
\hline Accommodation option & Number of rooms & Single & Double & Family options \\
\hline $\mathbf{1}$ & 46 & & $\mathrm{x}$ & \\
\hline $\mathbf{3}$ & 75 & & & $\mathrm{x}$ \\
\hline $\mathbf{4}$ & 37 & $\mathrm{x}$ & $\mathrm{x}$ & $\mathrm{x}$ \\
\hline $\mathbf{5}$ & 54 & $\mathrm{x}$ & $\mathrm{x}$ & \\
\hline Total & 144 & $\mathrm{x}$ & $\mathrm{x}$ & $\mathrm{x}$ \\
\hline
\end{tabular}

Table 1: Format of accommodation facilities in the SLHD

The SLHD established a comprehensive governance structure (Figure2) for the SHA to ensure both medical and Public Health matters were managed appropriately. The managers at each site report to the General Manager of the SHA, who is responsible for the planning, directing and ongoing management of the operations. Medical governance is provided by the A/Clinical Director of rpavirtual, the 24/7 virtual hospital service in the SLHD. There is a high level of oversight from the Chief Executive (CE) of the SLHD regarding decisions made within the service. This includes advice regarding appropriate selection and allocation of accommodation, initial investigations for those that become medically unstable and for all patient discharges.

\section{Site management}

As outlined in Figure 2, health care workers are on site 24/7 in the SHA. At least two registered nurses (RN) and two assistants-in-nursing (AIN) are present on site for health care needs. Additional staff include: the Site Manager, Accommodation Assistant, receptionist, cleaning staff and the building manager. Allied health staff on site consist of dieticians, social workers, psychologists, physiotherapists and speech pathology staff. They are able to cater to the varied needs of the residents as they arise.

All staff wear appropriate PPE and their designation is indicated by a sticker so that all patients are aware of the staff they may interact with. Any staff that enter the site are screened for COVID-19 risk factors and not allowed entry if they screen positive. Additional security is provided through the NSW Police to protect staff, the patients and the community in the event of patients not complying with quarantine requirements.

\section{Clinical management}


Clinical management of patients who are positive for COVID-19 occurs through rpavirtual and the SLHD Public Health Unit (PHU). rpavirtual is a virtual hospital located on SLHD's Royal Prince Alfred Hospital campus. This service provides remote, around-the-clock care for COVID-19 and other patients. It is supported by RPA Hospital specialists with an on-call roster for all sub-specialties. The joint management by clinical teams and the PHU is to ensure that patients' clinical needs are met and that appropriate Public Health Guidelines for isolation and clearance testing are applied to each case following their diagnosis. rpavirtual supports both the physical and mental health of these patients, and also provides advice for their families. Soon after implementation of virtual care for COVID-19 patients and in response to the levels of stress and anxiety displayed by patients in SHA quarantine, rpavirtual introduced a roster of psychologists and social workers. If any patient in the accommodation service becomes medically unstable and requires an escalation of their care this is co-ordinated with the rpavirtual Clinical Director, the PHU, NSW Ambulance service and the Emergency Department of Royal Prince Alfred Hospital.

Welfare checks are undertaken twice daily by qualified nursing staff. These take place through phone call and text messaging, dependent upon the preference of the patient. If there is no reply from the patient then this is escalated to a room check to ensure they are well. Adjustment to the loss of individual autonomy and uncertainty around their individual health has meant that a large range of issues are managed by these staff. The rare episodes of anger are escalated through security as required, and questioning of quarantine requirements are locally managed and then escalated to the Public Health Unit as required.

Discharge management of all persons in the SHA occurs in conjunction with the PHU. Each person's individual circumstances are reviewed through the PHU to ensure that their clinical needs have been met, and that they are following the appropriate Public Health Orders. This has involved communications with the NSW Ministry of Health and other jurisdictions within Australia, as many people in the SHA do not live within the Sydney Metropolitan area. This has required co-ordination with both clinical and public health services in their relevant jurisdictions for them to be able to return to their homes given the current varying restrictions that exist within Australia.

\section{Results}

\section{Demographics}

Data reported represents the accommodation occupancy from 29 March 2020 to 29 April 2020. The total patient number in the SHA during this time period was 520 , with 464 patients returned travellers, and 56 community patients from the SLHD. Community patients in the SHA consisted of people from within the SLHD and those from other sources such as cruise ships. The median age of patients in the SHA service was 35 , ranging from 2 months to 86 years old. Forty-six patients were children aged less than 18 years of age. There was an even distribution of gender with 182 female and 191 male patients. Returning travellers came from 5 international regions. The majority returned from North America $34 \%(n=128)$ and Europe $30 \%$ $(n=113)$. In this timeframe cumulatively 9661 people were resident in the accommodation service managed by SHEOC.

\section{Person Flow}

A total of 373 people were admitted to the SHA directly from the airport. Of these 337 were symptomatic and tested for COVID-19, the remaining 36 were accompanying family members. From those who were swabbed $88(26.1 \%)$ were positive for SARS-CoV 2 , representing $0.91 \%$ of all returning air travellers to NSW in this time period. During the same period 902 people (9.3\% of occupancy) in the SHEOC Accommodation were swabbed and 76 people were transferred from here to the Special Health Accommodation during their 14-day quarantine period due to need for medical care or a positive COVID-19 swab result. Of the positive cases in the SHA the day of positive diagnosis varied from Day 1 to Day 13 of their quarantine period. $63.6 \%(n=56)$ of these were diagnosed positive in the first week of quarantine.

Fifty per-cent of people in SHA were referred to rpavirtual for ongoing clinical care, whether due to their positive status, or the need for other medical or allied health assistance. Seven of the patients who tested positive for COVID-19 became medically unstable and required admission to RPA Hospital for ongoing medical care. The distribution of positive results by arrival location is shown in table 2 .

During this time frame 284 patients were discharged from the accommodation. The average length of stay in the SHA was 14.86 days (not including patients exempt from quarantine in NSW). The total quarantine length in any accommodation for patients was 16.17 days (including SHEOC accommodation stay). The average length of stay for the 72 discharged positive patients up to 30 April 2020 was 19.21 days (14-28 days).

The discharge address location for the patients varied. $58.4 \%(n=218)$ returned to a NSW address and the rest to interstate addresses with the majority to Queensland $19 \%(n=70)$ and $9 \%(n=32)$ from Victoria.

\section{Incidents}

An estimated $20 \%$ of patients reported concerns about their quarantine period to the SHA management. There were a total of 29 formal incidents notified through the internal SLHD system in this time-14 complaints, 6 security, 5 accident/ occupational health and safety incidents, 3 aggression and 1 fall. On site police were involved in 5 incidents due to aggression from the patients that required escalation. 


\begin{tabular}{|lllll|}
\hline & \multicolumn{5}{c|}{ COVID-19 Status } \\
\hline Region of origin & Negative & Not swabbed & Positive & Grand Total \\
\hline Asia & 29 & 6 & 7 & 42 \\
\hline Europe & 73 & 14 & 27 & 114 \\
\hline North America & 91 & 4 & 33 & 128 \\
\hline Oceania & 41 & 10 & 1 & 52 \\
\hline South America & 16 & 2 & 19 & 37 \\
\hline Grand Total & $\mathbf{2 5 0}$ & $\mathbf{3 6}$ & $\mathbf{8 7}$ & $\mathbf{3 7 3}$ \\
\hline
\end{tabular}

Table 2: COVID-19 status in SHA by region of origin 29 March - 29 April 2020

\section{Discussion}

Australia's COVID-19 pandemic response has posed unique challenges as well as opportunities for containment in the island nation. Australia has not faced a challenge to its population that has required this since the Influenza outbreak in 1919 (2). Australia's COVID-19 Public Health approach has been based on containment, as there is currently no effective vaccination or specific therapeutic options. As COVID-19 is associated with asymptomatic transmission and mild symptoms in many cases, multiple Public Health measures are required to decrease transmission in the community. Containment strategies such as quarantine, isolation, early case detection through testing and contact tracing, restriction of gatherings and implementation of social distancing are aimed at reducing person to person transmission as well as fomite transfer.(11) Modelling data which predicted the potential numbers of cases, deaths and impact on health service capacity informed the decisions to implement strong containment strategies and in particular, restrictive border measures, first with restriction of travel from China and then more broadly when analysis of transmission identified travel from other countries and cruise ships as factors introducing the epidemic in Australia.(12-14) Australian modelling studies had shown that in order to mitigate disease spread in Australia, isolation, quarantine and social distancing were required to ensure that Intensive Care Units (ICU) and the hospital system more broadly were not overwhelmed by a rapid increase in COVID-19 cases.(15-17).

The establishment of the SHA meant that quarantine isolation and management of suspected and confirmed cases, as well as management of close contacts could be comprehensively carried out for the returned traveller group. In NSW prior to the introduction of self-isolation for all returning travellers, notified cases were rapidly increasing, with 58\% comprised of returned travellers or their contacts up to 30 April 2020 . After the closure of Australia's borders on 20 March 2020, there was a gradual decrease in case numbers that were further reduced by the introduction of mandatory hotel quarantine on 29 March. Community transmission was also shown to decrease after this time.(18) Thus quarantine and isolation of returned travellers was a vital part of the Public Health response in mitigating COVID-19 spread in NSW and Australia.

The ability to monitor the health and wellbeing of the patients in the accommodation also meant that early case detection was able to be improved amongst an at-risk population. Australia has one of the highest testing rates per person worldwide for COVID-19, and this has been a vital part of containing spread of disease. The availability of testing within the SHA and the accommodation facilities managed by SHEOC throughout the quarantine period meant that new cases could be identified, isolated in an appropriate facility and managed clinically, as well as prevented ensuring separation from any accompanying at risk family members. Isolation of confirmed cases of COVID-19 until they had been clinically cleared by rpavirtual and the PHU meant that both the needs of the individual and the community were managed. The small number of people that required in hospital care returned to the SHA after discharge for any ongoing quarantine or clearance requirements after their admission.

Establishing a health service with multiple stakeholders and an evolving public health policy environment "on the run" was not without difficulty. The requirement to mobilise and provide a quarantine accommodation service required rapid recruitment and organisational management from NSW Health, NSW Police and the SLHD. Co-ordination and consistency of the aims of the accommodation was needed to follow the appropriate Public Health Orders as well as providing a high standard of clinical care for any unwell patients. At times, issues were identified only when problems or challenges arose, requiring a flexible, dynamic approach to management.

The ability to cater for individual needs was bound by the legal responsibility to ensure that the Public Health Orders were appropriately applied to every individual in the service in order to protect the needs of the community. Compulsory quarantine and isolation ensured that the welfare of the community was prioritised, however balancing the need of the individual and the community was challenging. The heterogeneity of the patients within the SHA and their varying needs required adaptability and daily review of all patients to ensure that their individual needs were met.

This was one of the challenging aspects of introducing and managing the SHA. SHA management estimated that $20 \%$ of patients escalated concerns regarding their quarantine period, however it is likely the true number is at least $50 \%$ due to the frequency of patients raising concerns with onsite staff that were not required to be escalated to more senior managers. Compliance with quarantine in previous International examples has varied significantly, and the number of those questioning their quarantine period potentially reflects those that would not adhere appropriately in the community. 
Understandably, individuals wished to advocate for themselves and their families regarding the conditions of their stay and these concerns needed to be adequately and sensitively addressed. According to the SHA management team, those that challenged their quarantine had four broad reasons for questioning their requirements to follow the Public Health Order. Unfortunately, many patients became verbally aggressive and emotional in this process.

- Poor understanding of risk for themselves and the community led to many people questioning why they needed to follow the Public Health Order, requiring further detailed discussion. The degree of illness experienced was influential - whether they were symptomatic and requiring a swab, negative or a case that was diagnosed as positive. Those that had mild symptoms seemed more likely to question the need to comply with the Public Health orders and to express dissatisfaction with their care.

- Some people understood the risks but formed the opinion that the risk did not apply to them.

- Some questioned the ethics and legality of the Public Health orders as they had been written and the application of them to their individual situation.

- Many people also found quarantine challenging to maintain due to their personal or family circumstances.

The importance of clear and consistent communication, and the provision of information has been shown to be important for the wellbeing of those adhering to quarantine.(19) When queries and concerns were raised, decision makers had the dual pressures of ensuring a timely and appropriate response for staff and patients, and navigating the potential ongoing implications of any decision made on the service and on the community. As the service has evolved, communication channels have become better understood and managed. The clear governance structure and delineation of roles within the management of the service allowed any issue that arose to be managed in a co-ordinated fashion, involving the General Manager of the service, the Public Health Unit, rpavirtual and the Chief Executive of the SLHD.

\section{Conclusion}

We have described the evolution of a unique health and accommodation service that required rapid recruitment and organisational management from NSW Health, NSW Police, Health Care Australia and the SLHD. This occurred in response to the need to follow the appropriate Public Health Orders while providing a high standard of clinical care for unwell patients. The current Public Health orders that exist in NSW and around Australia restricting the freedom of movement of individuals have not been in place in living memory for the majority of the population.

The SHA is a novel and important demonstration of the application of the Public Health principle of quarantine in contributing to the containment of COVID-19 in NSW, Australia. The learning from this process of establishment and management of the service is invaluable for any future disease outbreak requiring widespread quarantine and isolation in the population.

Ongoing review of the SHA is being undertaken to ensure that improvements to the service can be made for patients, staff and management. Long term follow up of the patients is planned to evaluate the impact of the quarantine on the health and social circumstances of the patients and staff involved in the service.

\section{Declarations}

\section{Acknowledgments}

Dr Ruth Armstrong

Imogen Baker

Yashoda Dhakal

Dr Edwina Dorney

Priyanka Gehlot

Sarah Marmara

Corryn McKay

Susan Pearce

\section{Competing interests}

The authors declare no competing interests. 


\section{Funding}

No external funding was given for this project or the production of this article

\section{References}

1.Barbisch D, Koenig KL, Shih F-Y. Is There a Case for Quarantine? Perspectives from SARS to Ebola. Disaster Med Public Health Prep . 2015 Oct;9(5):547-53. doi: 10.1017/dmp.2015.38. Epub 2015 Mar 23

2.Australian Bureau of Statistics. Human Quarantine: The Australian approach to a world problem. [23 November 2012; [cited 11 May 2020]; Available from: https://www.abs.gov.au/ausstats/abs@.nsf/featurearticlesbytitle/F74D29E8BE724723CA2569DE0024ED5C?OpenDocument.

3.Cumpston JHL. Influenza and Maritime Quarantine in Australia. National Library of Australia: Quarantine Service; Service Publication No.18, 1919

4.NSW Ministry of Health. NSW COVID-19 case statistics. 2020 [cited May 18 2020]; Available from:

https://www.health.nsw.gov.au/Infectious/covid-19/Pages/stats-nsw.aspx.

5.NSW Ministry of Health. Summary of the first 200 cases in NSW. 2020 [cited May 18 2020]; Available from:

https://www.health.nsw.gov.au/Infectious/covid-19/Pages/first-200-nsw-cases-summary.aspx

6.Wilder-Smith A, Freedman DO. Isolation, quarantine, social distancing and community containment: pivotal role for old-style public health measures in the novel coronavirus (2019-nCoV) outbreak. Journal of Travel Medicine. 2020;27(2). https://doi.org/10.1093/jtm/taaa020

7.Nussbaumer, Streit B, Mayr V, Dobrescu Al, Chapman A, Persad E, Klerings I, et al. Quarantine alone or in combination with other public health measures to control COVID-19: a rapid review. Cochrane Database of Systematic Reviews 2020, Issue 4. Art. No.: CD013574. DOI: 10.1002/14651858.CD013574

8.NSW Ministry of Health. Public Health (COVID-19 Quarantine) Order 2020 [cited 20 April 2020]; Available from: https://gazette.legislation.nsw.gov.au/so/download.w3p?id = Gazette_2020_2020-49.pdf

9.NSW Government. Public Health Orders (COVID-19 Air Transportation Quarantine) 2020 [cited 28 April 2020]; Available from: https://www.legislation.nsw.gov.au/_emergency/Public\%20Health\%20(COVID-19\%20Air\%20Transportation\%20Quarantine)\%200rder\%202020.pdf

10.NSW Government. Public Health (COVID-19 Maritime Quarantine) Order. 2020. [cited 28 April 2020] Accessible at:

https://www.legislation.nsw.gov.au/_emergency/Public\%20Health\%20(COVID19\%20Maritime\%20Quarantine)\%200rder\%202020_as\%20amended.pdf

11.Dalton CB, Corbett SJ, Katelaris AL. COVID-19: implementing sustainable low cost physical distancing and enhanced hygiene. Perspectives: Medical Journal of Australia (published online 05 May 2020). doi: 10.5694/mja2.50602

12.Linka K, Peirlinck M, Sahli Costabal F, Kuhl E. (2020) Outbreak dynamics of COVID-19 in Europe and the effect of travel restrictions, Computer Methods in Biomechanics and Biomedical Engineering, DOI: 10.1080/10255842.2020.1759560

13.Lee VJ, Chiew CJ, Khong WX. Interrupting transmission of COVID-19: lessons from containment efforts in Singapore, Journal of Travel Medicine, $202027(3)$ https://doi.org/10.1093/jtm/taaa039

14.Wells CR, Sah P, Moghadas SM, Pandey A, Shoukat A, Wang Y, et al. Impact of international travel and border control measures on the global spread of the novel 2019 coronavirus outbreak. Proceedings of the National Academy of Sciences Mar 2020, 117 (13) 7504-7509; DOI: $10.1073 /$ pnas. 2002616117

15.Webster RK, Brooks SK, Smith LE, Woodland L, Wessely S, Rubin GJ. How to improve adherence with quarantine: rapid review of the evidence. Public Health.182:163-9. doi: 10.1016/j.puhe.2020.03.007

16.Moss R. Modelling the impact of COVID-19 in Australia to inform transmission reducing

measures and health system preparedness. [Pre-published article]. In press 2020.

17.Shearer FM Walker J, Tellioglu N, McCaw JM, McVernon J,, Black A GN. Assessing the risk of spread of COVID-19 to the Asia Pacific region. The Peter Doherty Institute for Infection and Immunity [Pre-published article] In press 2020.

18.NSW Ministry of Health. COVID-19 weekly surveillance in NSW - Report for week ending April 302020 [cited May 62020 ] Accessible at: https://www.health.nsw.gov.au/Infectious/covid-19/Documents/covid-19-surveillance-report-20200430.pdf

19.Brooks SK, Webster RK, Smith LE, Woodland L, Wessely S, Greenberg N, et al. The psychological impact of quarantine and how to reduce it: rapid review of the evidence. The Lancet. 2020 395(10227) 912-920 https://doi.org/10.1016/S0140-6736(20)30460-8

Page $7 / 9$ 
Figures

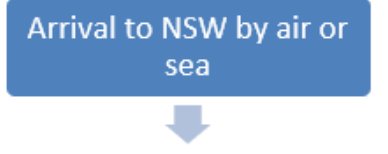

NSW Health screening of

all International Arrivals

Symptomatic travellers

swabbed for COVID-19

Sent to SHA

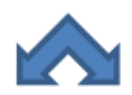

\section{Positive Result}

*

Allocation of long term accommodation

*

Referred to rpavirtual care

$\sqrt{9}$

Complete $\geq 14$ days
quarantine

Medically discharged by rpavirtual and Public Health

\section{Negative Result}

\begin{tabular}{|c|}
\hline $\begin{array}{c}\text { Allocation of long term } \\
\text { accommodation }\end{array}$ \\
\hline $\begin{array}{c}\text { Complete } \geq 14 \text { days } \\
\text { quarantine }\end{array}$ \\
\hline $\begin{array}{c}\text { Symptomatic patients } \\
\text { screened for COVID-19 }\end{array}$ \\
\hline Positive result - referred to \\
rpavirtual care
\end{tabular}

\section{Figure 1}

Referral flow chart for Returning International Travellers to NSW Special Health Accommodation 


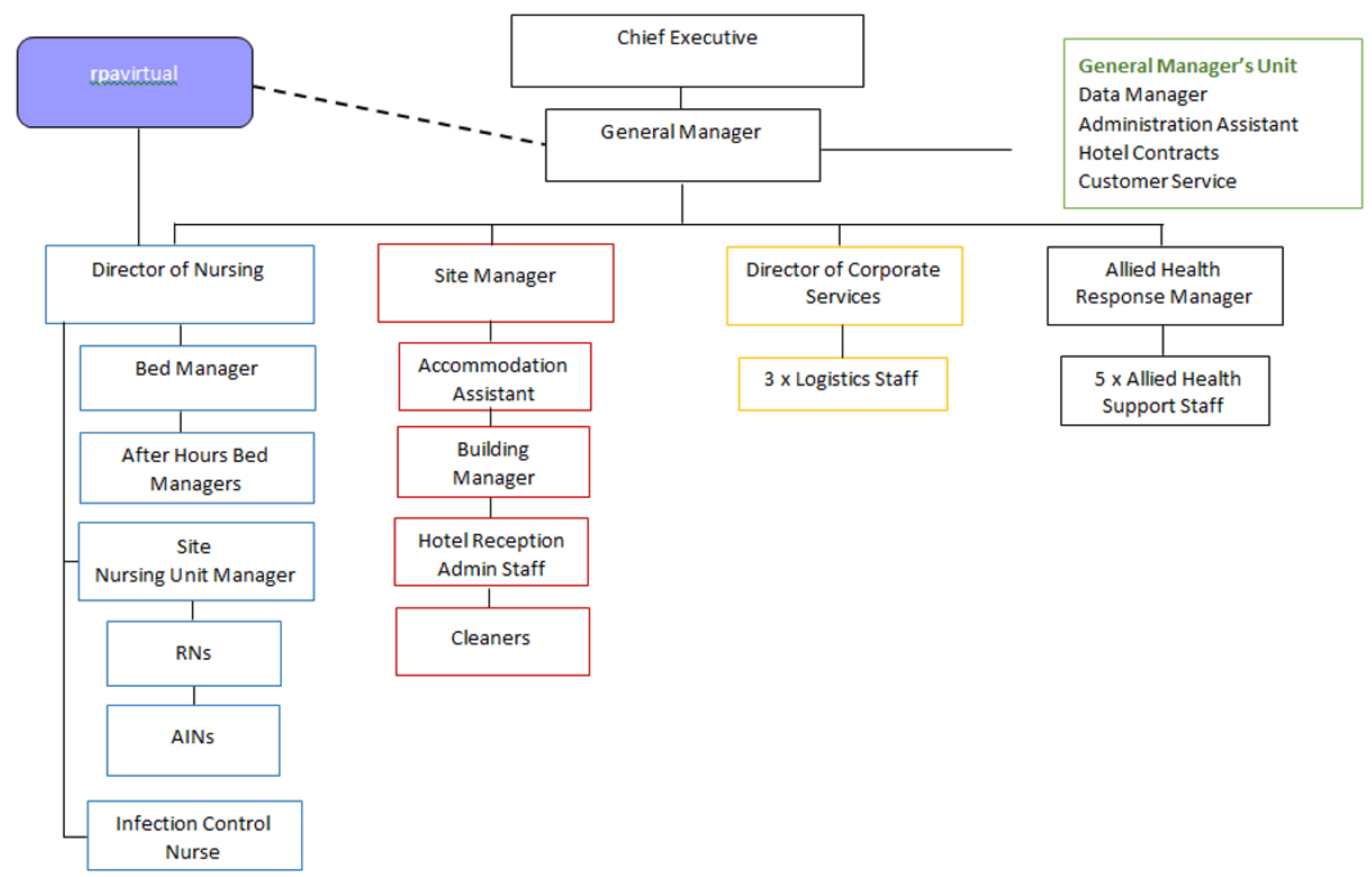

Figure 2

Clinical Governance Structure of the Special Health Accommodation 